mortality, outpatient mortality up to six months after discharge and hospital re-admission rates in the six months post discharge. Chi-squared test was applied to assess the relationship between respiratory acidosis and our outcomes.

Results 234 patients had an admission ABG and were subsequently followed up to the point of death or six months post discharge. Patients with a $\mathrm{PaCO}_{2}$ of $>6 \mathrm{Kpa}$ were 2.33 times (95\% CI 1.11 to 4.96 ) more likely to die in hospital as compared to those patients with a normal value. Patients with a lower arterial $\mathrm{pH}(<7.35)$ were 2.32 times $(95 \%$ CI 1.07 to 4.96) more likely to die in hospital as compared to those with a $\mathrm{pH}$ of $>7.35$. The increased risk in mortality was only seen for in-hospital mortality and there was no association with death in the 6 months following discharge, hospital re-admission or readmission for a respiratory problem.

Conclusion This data supports previous studies that suggest hypercapnia and respiratory acidosis are associated with increased inpatient mortality, therefore further demonstrating the usefulness of $\mathrm{pH}$ and $\mathrm{PaCO}_{2}$ as prognostic markers for inpatient outcomes. However our study does suggest that patients with respiratory acidosis on admission, who survive until discharge from hospital, do not have an increased risk of six month mortality or readmission compared to those with a normal admission ABG.

\section{P45 PRACTICAL USE OF THE DECAF SCORE: CAN WE IMPROVE OUTCOMES IN ACUTE EXACERBATION OF COPD ADMISSIONS?}

L Collier, T David, C Craig, R Yadavilli. Royal Bolton Hospital, Bolton, UK

\subsection{6/thoraxjnl-2015-207770.182}

Background Acute exacerbations of COPD (AECOPD) are the second most common cause of emergency hospital admission in England and are associated with an inpatient mortality rate of 4.3\%. ${ }^{1}$ The Dyspnoea, Eosinopenia, Consolidation, Acidaemia and Atrial Fibrillation (DECAF) Score, is an effective prognostic tool that predict mortality in AECOPD admissions. This scoring system is easy to apply during admission and has performed better than existing prognostic tools. ${ }^{2}$ We aim to appraise the efficacy of DECAF score in our busy respiratory and medical admissions unit.

Method Hospital admissions with AECOPD from Dec 2014 to Mar 2015 are prospectively reviewed and DECAF score applied to each patient. Morbidity and mortality indicators were then correlated with both total DECAF scores and each predictive index.

Results 78 admissions were reviewed, 60\% were male and the mean age was 72.7 years. Average length of stay was 15.3 days and 12 patients died in hospital. Our results were comparable with previous studies ${ }^{3}$, with inpatient mortality highest in those with DECAF scores of 3-5 (92\%) and lowest in those with scores of $0-1(0 \%)$. Higher DECAF scores were also associated with use of non-invasive ventilation (43\%).

Furthermore, each individual predictive index within the DECAF score was independently related to an increased mortality rate. There was $44 \%$ mortality in patients with atrial fibrillation and $30 \%$ mortality in patients with dyspnoea score of eMRC 5B. In-hospital mortality rate increased with each DECAF score (Figure 1).

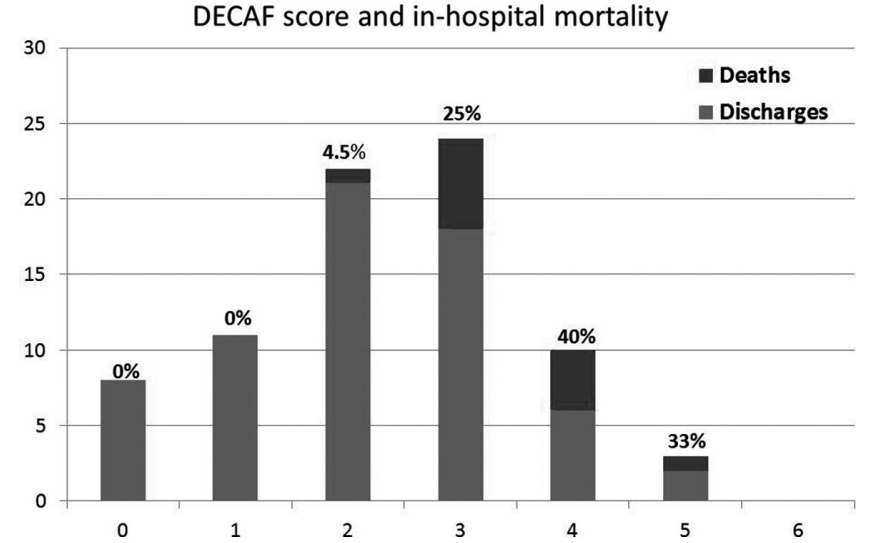

Abstract P45 Figure 1

Conclusions Introduction of DECAF score as clinical prediction tool for AECOPD admissions in our departments may be beneficial in reducing morbidity and mortality. Those scoring highest should be considered for early escalation, higher level of care and or palliative management. Those with lower scores may be suitable for early supported discharge. Further study of a larger group however is advisable to confirm the significance of these findings.

\section{REFERENCES}

1 National Chronic Obstructive Pulmonary Disease Audit Programme: Clinical audit of COPD exacerbations admitted to acute units in England and Wales, 2014

2 J Steer, J Gibson, S Bourke. The DECAF Score: predicting hospital mortality in exacerbations of chronic obstructive pulmonary disease. Thorax 2012;67:970-976

\section{P46 FREQUENCY OF COPD EXACERBATIONS IN THE GERMAN DACCORD REGISTRY}

${ }^{1} \mathrm{P}$ Kardos, ${ }^{2} \mathrm{R}$ Buhl, ${ }^{3} \mathrm{C}-\mathrm{P}$ Criée, ${ }^{4} \mathrm{C}$ Vogelmeier, ${ }^{5} \mathrm{C}$ Mailaender, ${ }^{6} \mathrm{H}$ Worth. ${ }^{1}$ Group Practice and Centre for Allergy, Respiratory and Sleep Medicine, Red Cross Maingau Hospita, Frankfurt, Germany; '2Pulmonary Department, Mainz University Hospital, Frankfurt, Germany; ${ }^{3}$ Department of Sleep and Respiratory Medicine, Evangelical Hospital GoettingenWeende, Bovenden, Germany: ${ }^{4}$ Department of Respiratory Medicine, University of Marburg, Marburg, Germany; ${ }^{5}$ Novartis Pharma GmbH, Nuremberg, Germany; ${ }^{6}$ Facharztforum Fuerth, Fuerth, Germany

\subsection{6/thoraxjnl-2015-207770.183}

Introduction In patients with COPD, exacerbations are among the most relevant safety measures. In this analysis of data from the observational DACCORD study, we report the frequency of exacerbations in a COPD population.

Methods To get insights into occurrence and frequency of exacerbations, data from 4,123 patients were obtained from 349 primary and secondary care centres in Germany. To be eligible for entry into DACCORD, all patients had to have a COPD diagnosis (consistent with the German Disease Management Programme definition), and had to have a change in bronchodilator maintenance medication, prior to entry. Data collected included history and treatment of exacerbations 6 months prior to inclusion, and for the duration of follow-up. Exacerbations were defined based on prescription of oral corticosteroids and/or antibiotics or on hospitalisation.

Results Mean age of the patients was 65.7 years; 36.9\% of patients had severe or very severe airflow limitation (GOLD $2010)$. In the 6 month period prior to study inclusion, $26.4 \%$ of the patients had at least one exacerbation. Fewer patients in the subgroup with CAT30 (16.7\% vs $47.9 \%$ ). Interestingly, $45 \%$ of 